DESY $97-074$

NTZ 14/97

hep-ph/9705264

April 1997

Phys. Lett. B in print

\title{
On the Evolution Kernels of Twist 2 Light-Ray Operators for Unpolarized and Polarized Deep Inelastic Scattering
}

\author{
Johannes Blümlein ${ }^{a}$, Bodo Geyer $^{b}$, and Dieter Robaschik ${ }^{a}$, \\ a DESY - Zeuthen, \\ Platanenallee 6, D-15735 Zeuthen, Germany \\ ${ }^{b}$ Naturwissenschaftlich-Theoretisches Zentrum der Universität Leipzig, \\ Augustusplatz 10, D-04109 Leipzig, Germany
}

\begin{abstract}
The non-singlet and singlet evolution kernels of the twist-2 light-ray operators for unpolarized and polarized deep inelastic scattering are calculated in $\mathcal{O}\left(\alpha_{s}\right)$ for the general case of virtualities $q^{2}, q^{2} \neq 0$. Special cases as the kernels for the general single-variable evolution equation and the Altarelli-Parisi and Brodsky-Lepage limits are derived from these results.
\end{abstract}




\section{Introduction}

The study of the Compton amplitude for scattering a virtual photon off a hadron is one of the basic tools in QCD to understand the short-distance behavior of the theory. The Compton amplitude for the general case of non-forward scattering is given by

$$
T_{\mu \nu}\left(p_{+}, p_{-}, Q\right)=i \int d^{4} x e^{i q x}\left\langle p_{2}\left|T\left(J_{\mu}(x / 2) J_{\nu}(-x / 2)\right)\right| p_{1}\right\rangle,
$$

where $p_{+}=p_{2}+p_{1}, p_{-}=p_{2}-p_{1}=q_{1}-q_{2}, Q=\left(q_{1}+q_{2}\right) / 2, p_{1}+q_{1}=p_{2}+q_{2}$. The kinematics of the basic process is depicted schematically in figure 1.

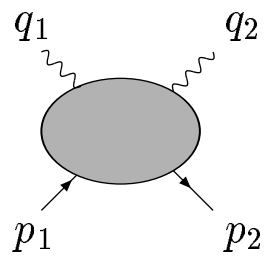

Figure 1: Kinematic notation for the Compton amplitude

The time-ordered product in eq. (1) can be represented in terms of the operator product expansion. Here we use the representation derived in ref. [1, 2],

$$
\begin{array}{r}
T\left(J_{\mu}(x / 2) J_{\nu}(-x / 2)\right) \approx \int_{-\infty}^{+\infty} d \kappa_{-} \int_{-\infty}^{+\infty} d \kappa_{+}\left[C_{a}\left(x^{2}, \kappa_{-}, \kappa_{+}, \mu^{2}\right) S_{\mu \nu}{ }^{\rho \sigma} \tilde{x}_{\rho} O_{\sigma}^{a}\left(\kappa_{+} \tilde{x}, \kappa_{-} \tilde{x}, \mu^{2}\right)\right. \\
\left.+C_{a, 5}\left(x^{2}, \kappa_{-}, \kappa_{+}, \mu^{2}\right) \varepsilon_{\mu \nu}{ }^{\rho \sigma} \tilde{x}_{\rho} O_{5, \sigma}^{a}\left(\kappa_{+} \tilde{x}, \kappa_{-} \tilde{x}, \mu^{2}\right)\right]
\end{array}
$$

with $S_{\mu \nu \rho \sigma}=g_{\mu \nu} g_{\rho \sigma}-g_{\mu \rho} g_{\nu \sigma}-g_{\mu \sigma} g_{\nu \rho}$ and $\varepsilon_{\mu \nu \rho \sigma}$ denoting the Levi-Civita symbol. The light-like vector

$$
\tilde{x}=x+r(x . r / r . r)\left[\sqrt{1-x \cdot x r \cdot r /(x \cdot r)^{2}}-1\right]
$$

is related to $x$ and a subsidiary four-vector $r . C_{a(5)}$ denote the respective coefficient functions. Unlike the local operator product expansion [3], which is mainly used in the case of forward scattering, the representation eq. (2) allows to derive the anomalous dimensions and coefficient functions also for the non-forward case for general values of the virtuality $q_{2}^{2}$.

In this paper we present the results of a calculation of the non-singlet and singlet twist $2^{1}$ anomalous dimensions for this general case both for unpolarized and polarized scattering. Details of the calculation will be published elsewhere [5]. We derive general evolution equations both for the operators and matrix elements in the non-forward case. Furthermore we derive a series of special cases discussed in the literature previously and compare the results.

\section{The Evolution Kernels}

We consider the flavor non-singlet and singlet operators in the axial gauge $\tilde{x}_{\mu} A^{\mu}=0$

$$
O^{\mathrm{NS}}\left(\kappa_{1}, \kappa_{2}\right)=\frac{i}{2}\left[\overline{\psi_{a}}\left(\kappa_{1} \tilde{x}\right) \lambda_{f} \gamma_{\mu} \tilde{x}^{\mu} \psi_{a}\left(\kappa_{2} \tilde{x}\right)-\overline{\psi_{a}}\left(\kappa_{2} \tilde{x}\right) \lambda_{f} \gamma_{\mu} \tilde{x}^{\mu} \psi_{a}\left(\kappa_{1} \tilde{x}\right)\right]
$$

\footnotetext{
${ }^{1}$ The notion of twist is not quite unambiguous in this context. It is rather used to label the type of operators being considered, for which in the limit $p_{2} \rightarrow p_{1}$ the notion applies [4].
} 


$$
\begin{aligned}
O_{5}^{\mathrm{NS}}\left(\kappa_{1}, \kappa_{2}\right) & =\frac{i}{2}\left[\overline{\psi_{a}}\left(\kappa_{1} \tilde{x}\right) \gamma_{5} \lambda_{f} \gamma_{\mu} \tilde{x}^{\mu} \psi_{a}\left(\kappa_{2} \tilde{x}\right)+\overline{\psi_{a}}\left(\kappa_{2} \tilde{x}\right) \gamma_{5} \lambda_{f} \gamma_{\mu} \tilde{x}^{\mu} \psi_{a}\left(\kappa_{1} \tilde{x}\right)\right] \\
O^{q}\left(\kappa_{1}, \kappa_{2}\right) & =\frac{i}{2}\left[\overline{\psi_{a}}\left(\kappa_{1} \tilde{x}\right) \gamma_{\mu} \tilde{x}^{\mu} \psi_{a}\left(\kappa_{2} \tilde{x}\right)-\overline{\psi_{a}}\left(\kappa_{2} \tilde{x}\right) \gamma_{\mu} \tilde{x}^{\mu} \psi_{a}\left(\kappa_{1} \tilde{x}\right)\right] \\
O_{5}^{q}\left(\kappa_{1}, \kappa_{2}\right) & =\frac{i}{2}\left[\overline{\psi_{a}}\left(\kappa_{1} \tilde{x}\right) \gamma_{5} \gamma_{\mu} \tilde{x}^{\mu} \psi_{a}\left(\kappa_{2} \tilde{x}\right)+\overline{\psi_{a}}\left(\kappa_{2} \tilde{x}\right) \gamma_{5} \gamma_{\mu} \tilde{x}^{\mu} \psi_{a}\left(\kappa_{1} \tilde{x}\right)\right] \\
O^{G}\left(\kappa_{1}, \kappa_{2}\right) & =\tilde{x}^{\mu} F_{a \mu}{ }^{\nu}\left(\kappa_{1} \tilde{x}\right) \tilde{x}^{\mu^{\prime}} F^{a}{ }_{\mu^{\prime} \nu}\left(\kappa_{2} \tilde{x}\right) \\
O_{5}^{G}\left(\kappa_{1}, \kappa_{2}\right) & =\frac{1}{2}\left[\tilde{x}^{\mu} F_{a \mu}{ }^{\nu}\left(\kappa_{1} \tilde{x}\right) \tilde{x}^{\mu^{\prime}} \tilde{F}^{a}{ }_{\mu^{\prime} \nu}\left(\kappa_{2} \tilde{x}\right)-\tilde{x}^{\mu} F^{a}{ }_{\mu \nu}\left(\kappa_{2} \tilde{x}\right) \tilde{x}^{\mu^{\prime}} \tilde{F}^{a}{ }_{\mu^{\prime}}{ }^{\nu}\left(\kappa_{1} \tilde{x}\right)\right],
\end{aligned}
$$

where $\psi_{a}$ denotes the quark field- and $F_{\mu \nu}^{a}$ the gluon field strength operators, respectively. The dual operator to $F_{\mu \nu}^{a}$ is

$$
\begin{gathered}
\tilde{F}_{\mu \nu}=\frac{1}{2} \varepsilon_{\mu \nu \rho \sigma} F^{\rho \sigma}, \\
\kappa_{1}=\kappa_{+}-\kappa_{-} \quad \kappa_{2}=\kappa_{+}+\kappa_{-},
\end{gathered}
$$

and $\lambda_{f}$ is a generator of the flavor group $S U\left(N_{f}\right), N_{f}$ being the number of active quark flavors.

The renormalization group equation implies the following evolution equations for these operators :

$$
\begin{aligned}
\mu^{2} \frac{d}{d \mu^{2}} O_{(5)}^{\mathrm{NS}}\left(\kappa_{1}, \kappa_{2}\right) & =\frac{\alpha_{s}\left(\mu^{2}\right)}{2 \pi} \int_{0}^{1} d \alpha_{1} \int_{0}^{1} d \alpha_{2} \theta\left(1-\alpha_{1}-\alpha_{2}\right) K^{\mathrm{NS}}\left(\alpha_{1}, \alpha_{2}\right) O_{(5)}^{\mathrm{NS}}\left(\kappa_{1}^{\prime}, \kappa_{2}^{\prime}\right), \\
\mu^{2} \frac{d}{d \mu^{2}}\left(\begin{array}{c}
O^{q}\left(\kappa_{1}, \kappa_{2}\right) \\
O^{G}\left(\kappa_{1}, \kappa_{2}\right)
\end{array}\right) & =\frac{\alpha_{s}\left(\mu^{2}\right)}{2 \pi} \int_{0}^{1} d \alpha_{1} \int_{0}^{1} d \alpha_{2} \theta\left(1-\alpha_{1}-\alpha_{2}\right) \boldsymbol{K}\left(\alpha_{1}, \alpha_{2}\right)\left(\begin{array}{c}
O^{q}\left(\kappa_{1}^{\prime}, \kappa_{2}^{\prime}\right) \\
O^{G}\left(\kappa_{1}^{\prime}, \kappa_{2}^{\prime}\right)
\end{array}\right),
\end{aligned}
$$

with $\alpha_{s}=g_{s}^{2} /(4 \pi)$ the strong coupling constant, $\mu$ the renormalization scale, and $\kappa_{1}^{\prime}=\kappa_{1}(1-$ $\left.\alpha_{1}\right)+\kappa_{2} \alpha_{1}, \kappa_{2}^{\prime}=\kappa_{2}\left(1-\alpha_{2}\right)+\kappa_{1} \alpha_{2}$. $\boldsymbol{K}$ denotes the matrix of the singlet evolution kernels in the unpolarized case. The singlet evolution equations for the polarized case are obtained replacing $O^{q, G}$ by $O_{5}^{q, G}$ and $\boldsymbol{K}$ by $\Delta \boldsymbol{K}$. As far as relations are concerned which are valid both for the unpolarized and polarized case under this replacement, we will, for brevity, only give that for the unpolarized case in the following. The matrices of the singlet kernels are

$$
\boldsymbol{K}=\left(\begin{array}{cc}
K^{q q} & K^{q G} \\
K^{G q} & K^{G G}
\end{array}\right) \quad \text { and } \quad \Delta \boldsymbol{K}=\left(\begin{array}{cc}
\Delta K^{q q} & \Delta K^{q G} \\
\Delta K^{G q} & \Delta K^{G G}
\end{array}\right)
$$

respectively. The non-singlet kernels obey $K^{\mathrm{NS}}=\Delta K^{\mathrm{NS}}=K^{q q}=\Delta K^{q q}$.

In the unpolarized case the kernels are given by

$$
\begin{aligned}
K^{q q}\left(\alpha_{1}, \alpha_{2}\right)= & C_{F}\left\{1-\delta\left(\alpha_{1}\right)-\delta\left(\alpha_{2}\right)+\delta\left(\alpha_{1}\right)\left[\frac{1}{\alpha_{2}}\right]_{+}+\delta\left(\alpha_{2}\right)\left[\frac{1}{\alpha_{1}}\right]_{+}+\frac{3}{2} \delta\left(\alpha_{1}\right) \delta\left(\alpha_{2}\right)\right\} \\
K^{q G}\left(\alpha_{1}, \alpha_{2}\right)= & -2 N_{f} T_{R} \kappa_{-}\left\{1-\alpha_{1}-\alpha_{2}+4 \alpha_{1} \alpha_{2}\right\} \\
K^{G q}\left(\alpha_{1}, \alpha_{2}\right)= & -C_{F} \frac{1}{\kappa_{-}-i \varepsilon}\left\{\delta\left(\alpha_{1}\right) \delta\left(\alpha_{2}\right)+2\right\} \\
K^{G G}\left(\alpha_{1}, \alpha_{2}\right)= & C_{A}\left\{4\left(1-\alpha_{1}-\alpha_{2}\right)+12 \alpha_{1} \alpha_{2}\right. \\
& \left.\quad+\delta\left(\alpha_{1}\right)\left(\left[\frac{1}{\alpha_{2}}\right]_{+}-2+\alpha_{2}\right)+\delta\left(\alpha_{2}\right)\left(\left[\frac{1}{\alpha_{1}}\right]_{+}-2+\alpha_{1}\right)\right\}+\frac{\beta_{0}}{2} \delta\left(\alpha_{1}\right) \delta\left(\alpha_{2}\right),
\end{aligned}
$$


in $\mathcal{O}\left(\alpha_{s}\right)$ where $C_{F}=\left(N_{c}^{2}-1\right) / 2 N_{c} \equiv 4 / 3, T_{R}=1 / 2, C_{A}=N_{c} \equiv 3, \beta_{0}=\left(11 C_{A}-4 T_{R} N_{f}\right) / 3$. The +-prescription is defined as

$$
\int_{0}^{1} d x[f(x, y)]_{+} \varphi(x)=\int_{0}^{1} d x f(x, y)[\varphi(x)-\varphi(y)],
$$

where the singularity of $f$ is of the type $\sim 1 /(x-y)$.

The kernels for the polarized case are

$$
\begin{aligned}
\Delta K^{q q}\left(\alpha_{1}, \alpha_{2}\right) & =K^{q q}\left(\alpha_{1}, \alpha_{2}\right) \\
\Delta K^{q G}\left(\alpha_{1}, \alpha_{2}\right) & =-2 N_{f} T_{R} \kappa_{-}\left\{1-\alpha_{1}-\alpha_{2}\right\} \\
\Delta K^{G q}\left(\alpha_{1}, \alpha_{2}\right) & =-C_{F} \frac{1}{\kappa_{-}-i \varepsilon}\left\{\delta\left(\alpha_{1}\right) \delta\left(\alpha_{2}\right)-2\right\} \\
\Delta K^{G G}\left(\alpha_{1}, \alpha_{2}\right) & =K^{G G}\left(\alpha_{1}, \alpha_{2}\right)-12 C_{A} \alpha_{1} \alpha_{2} .
\end{aligned}
$$

Whereas the kernels for the polarized case are derived for the first time, those for the unpolarized case were found several years ago already in refs. [6, []. The kernels $K^{i j}$ and $\Delta K^{i j}$ determine the respective evolutions of the operators $O_{(5)}^{\mathrm{NS}}, O_{(5)}^{q}$, and $O_{(5)}^{G}$ in $O\left(\alpha_{s}\right)$.

The evolution equations may also be written for the corresponding expectation values $\left\langle p_{2}\left|O_{(5)}^{q, G}\right| p_{1}\right\rangle$ to which two-variable quark and gluon partition functions are associated by

$$
\begin{aligned}
\frac{\left\langle p_{1}\left|O^{q}\right| p_{2}\right\rangle}{\left(i \tilde{x} p_{+}\right)}=e^{-i \kappa_{+} \tilde{x} p_{-}} \int_{-\infty}^{+\infty} d z_{+} \int_{-\infty}^{+\infty} d z_{-} e^{-i \kappa_{-}\left(\tilde{x} p_{+} z_{+}+\tilde{x} p_{-} z_{-}\right)} F_{q}\left(z_{-}, z_{+}\right) \\
\frac{\left\langle p_{1}\left|O^{G}\right| p_{2}\right\rangle}{\left(i \tilde{x} p_{+}\right)^{2}}=e^{-i \kappa_{+} \tilde{x} p_{-}} \int_{-\infty}^{+\infty} d z_{+} \int_{-\infty}^{+\infty} d z_{-} e^{-i \kappa_{-}\left(\tilde{x} p_{+} z_{+}+\tilde{x} p_{-} z_{-}\right)} F_{G}\left(z_{-}, z_{+}\right) .
\end{aligned}
$$

The evolution equations for the partition functions read

$$
\begin{aligned}
\mu^{2} \frac{d}{d \mu^{2}} F^{\mathrm{NS}}\left(z_{+}, z_{-}\right) & =\frac{\alpha_{s}\left(\mu^{2}\right)}{2 \pi} \int_{-\infty}^{+\infty} \frac{d z_{+}^{\prime}}{\left|z_{+}^{\prime}\right|} \int_{-\infty}^{+\infty} d z_{-}^{\prime} \widetilde{K}^{\mathrm{NS}}\left(\alpha_{1}, \alpha_{2}\right) F^{\mathrm{NS}}\left(z_{+}^{\prime}, z_{-}^{\prime}\right) \\
\mu^{2} \frac{d}{d \mu^{2}}\left(\begin{array}{c}
F^{q}\left(z_{+}, z_{-}\right) \\
F^{G}\left(z_{+}, z_{-}\right)
\end{array}\right) & =\frac{\alpha_{s}\left(\mu^{2}\right)}{2 \pi} \int_{-\infty}^{+\infty} \frac{d z_{+}^{\prime}}{\left|z_{+}^{\prime}\right|} \int_{-\infty}^{+\infty} d z_{-}^{\prime} \widetilde{\boldsymbol{K}}\left(z_{+}, z_{-} ; z_{+}^{\prime}, z_{-}^{\prime}\right)\left(\begin{array}{c}
F^{q}\left(z_{+}^{\prime}, z_{-}^{\prime}\right) \\
F^{G}\left(z_{+}^{\prime}, z_{-}^{\prime}\right)
\end{array}\right),
\end{aligned}
$$

where $F^{\mathrm{NS}}\left(z_{+}, z_{-}\right)=F^{q_{i}}\left(z_{+}, z_{-}\right)-F^{\bar{q}_{j}}\left(z_{+}, z_{-}\right)$, and

$$
\widetilde{K}^{i j}\left(\alpha_{1}, \alpha_{2}\right)=\frac{1}{2} \int_{0}^{1} d z_{+}^{\prime \prime} \widetilde{O}^{i j}\left(z_{+}, z_{+}^{\prime \prime}\right) \theta\left(1-\alpha_{+}^{\prime}\right) \theta\left(\alpha_{+}^{\prime}\right) \theta\left(\alpha_{+}^{\prime}+\alpha_{-}^{\prime}\right) \theta\left(\alpha_{+}^{\prime}-\alpha_{-}^{\prime}\right) K^{i j}\left(\alpha_{1}^{\prime}, \alpha_{2}^{\prime}\right),
$$

with $\widetilde{K}^{\mathrm{NS}}=\widetilde{K}^{q q}$ and $\alpha_{\rho}^{\prime}=\alpha_{\rho}\left(z_{+} \rightarrow z_{+}^{\prime \prime}\right)$,

$$
\begin{gathered}
\alpha_{1}=\frac{\alpha_{+}+\alpha_{-}}{2} \quad \alpha_{2}=\frac{\alpha_{+}-\alpha_{-}}{2}, \\
\alpha_{+}=1-\frac{z_{+}}{z_{+}^{\prime}} \quad \alpha_{-}=\frac{z_{+} z_{-}^{\prime}-z_{-} z_{+}^{\prime}}{z_{+}^{\prime}},
\end{gathered}
$$

and

$$
\widetilde{O}^{i j}\left(z_{+}, z_{+}^{\prime \prime}\right)=\left(\begin{array}{rr}
\delta\left(z_{+}-z_{+}^{\prime \prime}\right) & \partial_{z_{+}} \delta\left(z_{+}-z_{+}^{\prime \prime}\right) \\
-\theta\left(z_{+}-z_{+}^{\prime \prime}\right) & \delta\left(z_{+}-z_{+}^{\prime \prime}\right)
\end{array}\right)
$$




\section{Special Cases}

The evolution kernels given above cover a series of limiting cases which were studied before. These are characterized by special kinematic conditions for the matrix elements, as in the case of forward scattering $\left\langle p_{2}\right| \rightarrow\left\langle p_{1}\right| \equiv\langle p|$ or the transition from the vacuum state $\langle 0|$ to a hadron state $\langle p|$. These conditions lead to a constraint between the variables $z_{+}$and $z_{-}$, which is defined by a variable $t=\chi\left(z_{+}, z_{-}\right)$and implies related evolution equations for partition functions depending

on the variable $t$. These partition functions are related to the expectation values of the operators $Q^{q}$ and $O^{G}$ by

$$
\begin{aligned}
\left.\frac{\left\langle p_{1}\left|O^{q}\left(-\kappa_{-} \tilde{x}, \kappa_{-} \tilde{x}\right)\right| p_{2}\right\rangle}{\left(i \tilde{x} p_{+}\right)}\right|_{\tilde{x} p_{-}=\tau \tilde{x} p_{+}} & =\int_{-\infty}^{+\infty} d t e^{-i \kappa_{-} \tilde{x} p_{+} t} F_{q}(t) \\
\left.\frac{\left\langle p_{1}\left|O^{G}\left(-\kappa_{-} \tilde{x}, \kappa_{-} \tilde{x}\right)\right| p_{2}\right\rangle}{\left(i \tilde{x} p_{+}\right)^{2}}\right|_{\tilde{x} p_{-}=\tau \tilde{x} p_{+}} & =\int_{-\infty}^{+\infty} d t e^{-i \kappa_{-} \tilde{x} p_{+} t} t F_{G}(t) .
\end{aligned}
$$

Here $\tau=\tilde{x} p_{-} / \tilde{x} p_{+}$is a general parameter which distinguishes the cases discussed below.

The evolution equations are :

$$
\begin{aligned}
\mu^{2} \frac{d}{d \mu^{2}} F^{\mathrm{NS}}(t) & =\frac{\alpha_{s}\left(\mu^{2}\right)}{2 \pi} \int_{-\infty}^{+\infty} d t^{\prime} V_{e x t}^{\mathrm{NS}}\left(t, t^{\prime}, \tau\right) F^{\mathrm{NS}}\left(t^{\prime}\right) \\
\mu^{2} \frac{d}{d \mu^{2}}\left(\begin{array}{c}
F^{q}(t) \\
F^{G}(t)
\end{array}\right) & =\frac{\alpha_{s}\left(\mu^{2}\right)}{2 \pi} \int_{-\infty}^{+\infty} d t^{\prime} \boldsymbol{V}_{\text {ext }}\left(t, t^{\prime}, \tau\right)\left(\begin{array}{c}
F^{q}\left(t^{\prime}\right) \\
F^{G}\left(t^{\prime}\right)
\end{array}\right),
\end{aligned}
$$

with $F^{\mathrm{NS}}=F^{q_{i}}-F^{\bar{q}_{j}}$ and $V^{\mathrm{NS}}=V^{q q}$. The corresponding extended kernel $\boldsymbol{V}\left(t, t^{\prime}, \tau\right)=$ $\left(V^{i, j}\left(t, t^{\prime}, \tau\right)\right)$ reads

$$
\begin{aligned}
V_{e x t}^{i j}\left(t, t^{\prime}, \tau\right)= & \int_{0}^{1} d \alpha_{1} \int_{0}^{1-\alpha_{1}} d \alpha_{2} K^{i j}\left(\alpha_{1}, \alpha_{2}\right) \frac{1}{2 \pi} \int_{-\infty}^{+\infty} d\left(p_{+} \tilde{x} \kappa_{-}\right) \\
& \times\left[i p_{+} \tilde{x}\left(\kappa_{-}-i \varepsilon\right)\right]^{a_{i j}} \frac{t^{\prime a_{j}}}{t^{a_{i}}} \exp \left\{i p_{+} \kappa_{-} \tilde{x}\left[t-\left(1-\alpha_{1} t^{\prime}-\alpha_{2}\right)+\tau\left(\alpha_{1}-\alpha_{2}\right)\right]\right\}
\end{aligned}
$$

with $a_{i j}=a_{j}-a_{i}, a_{G}=1, a_{q}=0$. It obeys the scaling relation

$$
V_{e x t}^{i j}\left(t, t^{\prime}, \tau\right)=\frac{1}{\tau} V_{e x t}^{i j}\left(\frac{t}{\tau}, \frac{t^{\prime}}{\tau}, 1\right) .
$$

For convenience we write the general expressions for the evolution kernels in the variables

$$
x=\frac{1}{2}\left(1+\frac{t}{\tau}\right), \quad y=\frac{1}{2}\left(1+\frac{t^{\prime}}{\tau}\right) .
$$

They are given by :

$$
\begin{aligned}
V_{e x t}^{q q}\left(t, t^{\prime}, \tau\right) & =\frac{1}{2}\left\{V^{q q}(x, y) \rho(x, y)+V^{q q}(\bar{x}, \bar{y}) \rho(\bar{x}, \bar{y})\right\} \frac{1}{\tau} \\
V_{e x t}^{q G}\left(t, t^{\prime}, \tau\right) & =\frac{1}{2}\left(\frac{2 y-1}{2}\right)\left\{V^{q G}(x, y) \rho(x, y)-V^{q G}(\bar{x}, \bar{y}) \rho(\bar{x}, \bar{y})\right\} \frac{1}{\tau} \\
V_{\text {ext }}^{G q}\left(t, t^{\prime}, \tau\right) & =\frac{1}{2}\left(\frac{2}{2 x-1}\right)\left\{V^{G q}(x, y) \rho(x, y)-\bar{V}^{G q}(\bar{x}, \bar{y}) \rho(\bar{x}, \bar{y})\right\} \frac{1}{\tau}
\end{aligned}
$$




$$
\begin{aligned}
V_{e x t}^{G G}\left(t, t^{\prime}, \tau\right) & =\frac{1}{2}\left(\frac{2 y-1}{2 x-1}\right)\left\{V^{G G}(x, y) \rho(x, y)+V^{G G}(\bar{x}, \bar{y}) \rho(\bar{x}, \bar{y})\right\} \frac{1}{\tau} \\
\Delta V_{e x t}^{q q}\left(t, t^{\prime}, \tau\right) & =V_{e x t}^{q q}\left(t, t^{\prime}, \tau\right) \\
\Delta V_{e x t}^{q G}\left(t, t^{\prime}, \tau\right) & =\frac{1}{2}\left(\frac{2 y-1}{2}\right)\left\{\Delta V^{q G}(x, y) \rho(x, y)-\Delta V^{q G}(\bar{x}, \bar{y}) \rho(\bar{x}, \bar{y})\right\} \frac{1}{\tau} \\
\Delta V_{e x t}^{G q}\left(t, t^{\prime}, \tau\right) & =\frac{1}{2}\left(\frac{2}{2 x-1}\right)\left\{\Delta V^{G q}(x, y) \rho(x, y)-\Delta \bar{V}^{G q}(\bar{x}, \bar{y}) \rho(\bar{x}, \bar{y})\right\} \frac{1}{\tau} \\
\Delta V_{e x t}^{G G}\left(t, t^{\prime}, \tau\right) & =\frac{1}{2}\left(\frac{2 y-1}{2 x-1}\right)\left\{\Delta V^{G G}(x, y) \rho(x, y)+\Delta V^{G G}(\bar{x}, \bar{y}) \rho(\bar{x}, \bar{y})\right\} \frac{1}{\tau}
\end{aligned}
$$

with

$$
\rho(x, y)=\theta\left(1-\frac{x}{y}\right) \theta\left(\frac{x}{y}\right) \operatorname{sign}(y)
$$

and

$$
\begin{aligned}
V^{q q}(x, y) & =C_{F}\left[\frac{x}{y}-\frac{1}{y}-\frac{1}{(y-x)_{+}}+\frac{3}{2} \delta(x-y)\right] \\
V^{q G}(x, y) & =-2 N_{f} T_{R} \frac{x}{y}\left[4(1-x)+\frac{1-2 x}{y}\right] \\
V^{G q}(x, y) & =C_{F}\left[\frac{y^{2}-x^{2}}{y}+1\right] \quad \bar{V}^{G q}(x, y)=C_{F}\left[\frac{y^{2}-x^{2}}{y}-1\right] \\
V^{G G}(x, y) & =C_{A}\left[2 \frac{x^{2}}{y}\left(3-2 x+\frac{1-x}{y}\right)+\frac{1}{(y-x)_{+}}-\frac{y-x}{y^{2}}\right]+\frac{\beta_{0}}{2} \delta(x-y) \\
\Delta V^{q q}(x, y) & =V^{q q}(x, y) \\
\Delta V^{q G}(x, y) & =-2 N_{f} T_{R} \frac{x}{y^{2}} \\
\Delta V^{G q}(x, y) & =C_{F}\left[1-\frac{y^{2}-x^{2}}{y}\right] \\
\Delta V^{G G}(x, y) & =C_{A}\left[2 \frac{x^{2}}{y^{2}}+\frac{1}{(y-x)_{+}}-\frac{y-x}{y^{2}}\right]+\frac{\beta_{0}}{2} \delta(x-y)
\end{aligned}
$$

Note that the kernels given in eqs. (39 46) apply to the full range of variables. The function $V_{\text {ext }}^{q q}\left(t, t^{\prime}, \tau\right)$ was already derived in refs. [8, 2]. For the unpolarized case the kernels (39 42) were also calculated in ref. 99 recently, using a different notation. There separate expressions for different ranges of variables are given. For $\tau=1$, corresponding to $\zeta=1$ in [9], the kernels of [9] for $0 \leq x, y \leq 1$ agree with the result obtained above up to obvious misprints. Let us specify now the parameter $\tau$ to derive a series of different evolution kernels discussed in the literature.

\section{$3.1 \quad t^{\prime}=1$}

In this case, which has been dealt with in ref. [10 recently, the evolution kernels depend on one variable unlike the case discussed in the previous section. Furthermore the parameter $\tau$ introduced above equals to $-\xi / 2$ in the notation of ref. [10], and the value of the final state virtuality is chosen as $q_{2}^{2}=0$. Whereas we obtain the corresponding evolution kernels as a special limit from the general two-variable kernels eqs. (15-18, 20-23), these kernels were evaluated in [10] only in a restricted range of variables. We agree with the results given in [10] for $t>\xi / 2$, 
eqs. $(22,30) .^{2}$ These equations, generalizing them even for $t^{\prime} \neq 1$, read

$$
\begin{aligned}
K^{q q}\left(t, t^{\prime}, \xi\right) & =C_{F} \frac{t^{2}+t^{\prime 2}-\xi^{2} / 2}{\left(t^{\prime 2}-\xi^{2} / 4\right)\left(t^{\prime}-t\right)_{+}}+\frac{3}{2} \delta\left(t^{\prime}-t\right) \\
K^{q G}\left(t, t^{\prime}, \xi\right) & =T_{R} N_{f} \frac{t^{2}+\left(t^{\prime}-t\right)^{2}-\xi^{2} / 4}{\left(t^{\prime 2}-\xi^{2} / 4\right)^{2}} t^{\prime} \\
K^{G q}\left(t, t^{\prime}, \xi\right) & =C_{F} \frac{t^{\prime 2}+\left(t^{\prime}-t\right)^{2}-\xi^{2} / 4}{t\left(t^{\prime 2}-\xi^{2} / 4\right)} \\
K^{G G}\left(t, t^{\prime}, \xi\right)= & 2 C_{A}\left(\frac{t^{\prime}}{t}\right) \frac{1}{\left(t^{\prime 2}-\xi^{2} / 4\right)^{2}}\left[\frac{\left(t^{2}-\xi^{2} / 4\right)^{2}}{\left(t^{\prime}-t\right)_{+}}+t^{\prime}\left(t^{2}+\xi^{2} / 4\right)\right. \\
\Delta K^{q q}\left(t, t^{\prime}, \xi\right)= & K^{q q}\left(t, t^{\prime}, \xi\right) \\
\Delta K^{q G}\left(t, t^{\prime}, \xi\right)= & \left.\left.T_{R} N_{f} \frac{t^{2}-\left(t^{\prime}-t\right)^{2}-\xi^{2} / 4}{\left(t^{\prime 2}-\xi^{2} / 4\right)^{2}} t^{\prime} / 4\right)-\left(t^{\prime}+t\right)\left(t^{\prime}-t\right)^{2}\right]+\frac{\beta_{0}}{2} \delta\left(t^{\prime}-t\right), \\
\Delta K^{G q}(x, \xi)= & C_{F} \frac{t^{\prime}-\left(t^{\prime}-t\right)^{2}-\xi^{2} / 4}{t\left(t^{\prime 2}-\xi^{2} / 4\right)} \\
\Delta K^{G G}(x, \xi)= & 2 C_{A}\left(\frac{t^{\prime}}{t}\right) \frac{1}{\left(t^{\prime 2}-\xi^{2} / 4\right)^{2}}\left[\frac{\left(t^{\prime 2}-\xi^{2} / 4\right)^{2}}{\left(t^{\prime}-t\right)_{+}}+t^{\prime}\left(t^{\prime 2}+\xi^{2} / 4\right)\right. \\
& \left.-t\left(3 t^{\prime 2}-\xi^{2} / 4\right)-2 t^{\prime}\left(t^{\prime}-t\right)^{2}\right]+\frac{\beta_{0}}{2} \delta\left(t^{\prime}-t\right) .
\end{aligned}
$$

\subsection{The Brodsky-Lepage Limit}

For $\tau= \pm 1$ the equations (39 46) transform into the limit $\left\langle p_{2}\right| \rightarrow\langle p|,\left\langle p_{1}\right| \rightarrow\langle 0|$, which is known as the Brodsky-Lepage [12] and Efremov-Radyushkin [13 case. This limit may be performed formally leaving $p_{1} \rightarrow 0$, leading to correct results, cf. [8]. The corresponding evolution equations are obtained using as variables $x$ and $y$, eq. (38), in (34, 35).

\subsection{The Altarelli-Parisi Limit}

We consider the case of forward scattering $p_{2}=p_{1} \equiv p$. The corresponding evolution kernels can be obtained after some calculation from eqs. (39 46) in the limit $\tau \rightarrow 0$ or, alternatively, by a direct calculation in the forward scattering case using the kernels eqs. (15-23), which is performed in the following.

The quark and gluon distributions are related to the operator expectation values by

$$
f^{q}(z, \mu)=\frac{1}{2 \pi} \int_{-\infty}^{+\infty} d\left(2 p \tilde{x} \kappa_{-}\right)\left\langle p\left|O^{q}\right| p\right\rangle\left(\kappa_{-}, \mu\right) \frac{e^{2 i p \tilde{x} \kappa_{-}}}{2 i p \tilde{x}}
$$

\footnotetext{
${ }^{2} \mathrm{~A}$ deviating result was reported in ref. [11] recently studying the unpolarized case. However, as the kinematic quantities used are not specified in detail, a comparison is not possible. Note also typographical errors in the color factors.

${ }^{3}$ In ref. 10 the variable $t$ was denoted by $x$, having a different meaning in our notation. Therefore we use $t$ instead in eqs. 56 63).

${ }^{4}$ Several independent calculations of the evolution kernels for the meson wave functions were performed in refs. [14].
} 


$$
z f^{G}(z, \mu)=\frac{1}{2 \pi} \int_{-\infty}^{+\infty} d\left(2 p \tilde{x} \kappa_{-}\right)\left\langle p\left|O^{G}\right| p\right\rangle\left(\kappa_{-}, \mu\right) \frac{e^{2 i p \tilde{x} \kappa_{-}}}{(2 i p \tilde{x})^{2}}
$$

The respective polarized parton densities are obtained by replacing $f^{q, G}(z)$ by $\Delta f^{q, G}(z)$ and $O^{q, G}$ by $O_{5}^{q, G}$. The evolution equation for the operators $O_{(5)}^{q, G}$, eqs. (12, 13), can be rewritten in this limit by

$$
\begin{aligned}
\mu^{2} \frac{d}{d \mu^{2}} f^{\mathrm{NS}}(z, \mu) & =\frac{\alpha_{s}\left(\mu^{2}\right)}{2 \pi} \int_{-\infty}^{+\infty} \frac{d z^{\prime}}{\left|z^{\prime}\right|} \widehat{P}^{\mathrm{NS}}\left(\frac{z}{z^{\prime}}\right) f^{\mathrm{NS}}(z, \mu), \\
\mu^{2} \frac{d}{d \mu^{2}}\left(\begin{array}{c}
f^{q}(z, \mu) \\
f^{G}(z, \mu)
\end{array}\right) & =\frac{\alpha_{s}\left(\mu^{2}\right)}{2 \pi} \int_{-\infty}^{+\infty} \frac{d z^{\prime}}{\left|z^{\prime}\right|} \widehat{\boldsymbol{P}}\left(\frac{z}{z^{\prime}}\right)\left(\begin{array}{c}
f^{q}(z, \mu) \\
f^{G}(z, \mu)
\end{array}\right) .
\end{aligned}
$$

The splitting functions $\widehat{P}^{i j}(z)\left(\Delta \widehat{P}^{i j}(z)\right)$ are related to the kernels $K^{i j}\left(\alpha_{1}, \alpha_{2}\right)\left(\Delta K^{i j}\left(\alpha_{1}, \alpha_{2}\right)\right)$ by

$$
\begin{aligned}
& \widehat{P}^{i j}(z)=P^{i j}(z) \theta(z) \theta(1-z) \\
& P^{i j}(z)=\int_{-\infty}^{+\infty} d u \widehat{O}^{i j}(u, z) \int_{0}^{1} d \xi(1-u) \widehat{K}^{i j}\left(\alpha_{1}, \alpha_{2}\right) \theta(1-u) \theta(u),
\end{aligned}
$$

with $\alpha_{1}=\xi(1-u), \alpha_{2}=(1-\xi)(1-u)$, and

$$
\widehat{\boldsymbol{K}}=\left(\begin{array}{rr}
K^{q q} & \left(1 / \kappa_{-}\right) K^{q G} \\
\left(\kappa_{-}-i \varepsilon\right) K^{G q} & K^{G G}
\end{array}\right), \quad \widehat{O}^{i j}(u, z)=\left(\begin{array}{cr}
\delta(z-u) & \partial_{z} \delta(z-u) \\
-\theta(z-u) / z & \delta(z-u) / z
\end{array}\right)
$$

$P^{i j}(z)\left(\Delta P^{i j}(z)\right)$ are the well-known Altarelli-Parisi splitting functions [15] for unpolarized and polarized deep-inelastic scattering

$$
\begin{aligned}
P^{q q}(z) & =C_{F}\left(\frac{1+z^{2}}{1-z}\right)_{+} \\
P^{q G}(z) & =2 N_{f} T_{R}\left[z^{2}+(1-z)^{2}\right] \\
P^{G q}(z) & =C_{F} \frac{1+(1-z)^{2}}{z} \\
P^{G G}(z) & =2 C_{A}\left[\frac{1}{z}+\frac{1}{(1-z)}+2+z(1-z)\right]+\frac{\beta_{0}}{2} \delta(1-z), \\
\Delta P^{q q}(z) & =P^{q q}(z) \\
\Delta P^{q G}(z) & =2 N_{f} T_{R}\left[z^{2}-(1-z)^{2}\right] \\
\Delta P^{G q}(z) & =C_{F} \frac{1-(1-z)^{2}}{z} \\
\Delta P^{G G}(z) & =2 C_{A}\left[1-2 z+\frac{1}{(1-z)}+\right]+\frac{\beta_{0}}{2} \delta(1-z) .
\end{aligned}
$$

In deriving eq. (68) it is useful to apply the relations

$$
\theta(x)=\lim _{\varepsilon \rightarrow 0^{+}} \frac{1}{2 \pi} \int_{-\infty}^{+\infty} d \xi \frac{e^{i x \xi}}{i \xi+\varepsilon}, \quad \delta^{(k)}(x)=\frac{1}{2 \pi} \int_{-\infty}^{+\infty} d \xi(i \xi)^{k} e^{i x \xi}
$$

which are valid for tempered distributions 16. 


\section{Conclusions}

The evolution kernels for the twist 2 light-ray operators both for the case of unpolarized and polarized deep inelastic non-forward scattering were derived for the flavor non-singlet and singlet cases. In general the partition functions depend on two distribution variables. One may study as well specialized evolution equations in one distribution variable implying external constraints, covering the case of evolution equations for non-forward parton densities. In this way, among various others, also the well-known evolution equations as the Brodsky-Lepage or AltarelliParisi equations can be obtained.

Acknowledgement We would like to thank Paul Söding for his constant support of the project and D. Müller for discussions on the present topic.

\section{References}

[1] S.A. Anikin and O.I. Zavialov, Ann. Phys. (NY) 116 (1978) 135;

O.I. Zavialov, Renormalized Feynman Diagrams, (Nauka, Moscow, 1979), in Russian;

Renormalized Quantum Field Theory (Kluwer Academic Press, Dordrecht, 1990).

[2] D. Müller, D. Robaschik, B. Geyer, F.-M. Dittes, and J. Hořejši, Fortschr. Phys. 42 (1994) 2 .

[3] K.G. Wilson, Phys. Rev. 179 (1969) 1699:

R.A. Brandt and G. Preparata, Fortschr. Phys. 18 (1970) 249;

W. Zimmermann, Lect. on Elementary Particle Physics and Quantum Field Theory, Brandeis Summer Inst., Vol. 1, (MIT Press, Cambridge, 1970);

Y. Frishman, Ann. Phys. 66 (1971) 373;

N. Christ, B. Hasslacher, and A.H. Mueller, Phys. Rev. D6 (1972) 3543.

[4] D.J. Gross and S.B. Treiman, Phys. Rev. D4 (1971) 1059.

[5] J. Blümlein, B. Geyer, and D. Robaschik, in preparation.

[6] T. Braunschweig, B. Geyer, and D. Robaschik, Ann. Phys. (Leipzig) 44 (1987) 407.

[7] I. Balitzky and V. Braun, Nucl. Phys. B311 (1989) 541.

[8] F.-M. Dittes, D. Müller, D. Robaschik, B. Geyer, and J. Hořejši, Phys. Lett. B209 (1988) 325.

[9] A.V. Radyushkin, hep-ph/9704207.

[10] X. Ji, hep-ph/9609381.

[11] L. Frankfurt, A. Freund, V. Guzey, and M. Strikman, hep-ph/9703449.

[12] G.P. Lepage and S.J. Brodsky, Phys. Lett. B87 (1979)359; Phys. Rev. D22 (1980) 2157.

[13] A.V. Efremov and A.V. Radyushkin, Phys. Lett. 94B (1980) 245; Theor. Math. Phys. 42 (1980) 97. 
[14] M.K. Chase, Nucl. Phys. B174 (1980) 109;

T. Ohrndorf, Nucl. Phys. B186 (1981) 153;

M.A. Shifman and M.I. Vysotsky, Nucl. Phys. B186 (1981) 475;

M.V. Terentjev, Yad. Fiz. 33 (1981) 1692;

V.N. Baier and A.B. Grozin, Sov. J. Nucl. Phys. 35 (1982) 899; Nucl. Phys. B192 (1981) 476.

[15] D. Gross and F. Wilczek, Phys. Rev. D8 (1974) 416; D9 (1974) 980;

H. Georgi and D. Politzer, Phys. Rev. D9 (1974) 416;

L. Lipatov, Sov. J. Nucl. Phys. 20 (1975) 94;

G. Altarelli and G. Parisi, Nucl. Phys. B126 (1977) 278;

K. Kim and K. Schilcher, Phys. Rev. D17 (1978) 2800;

Yu. Dokshitser, Sov. Phys. JETP 46 (1977) 641;

H. Ito, Prog. Theor. Phys. 54 (1975) 555;

K. Sasaki, Progr. Theor . Phys. 54 (1975) 1816;

M.A. Ahmed and G.G. Ross, Phys. Lett. B56 (1975) 385; Nucl. Phys. B111 (1976) 298.

[16] V.S. Vladimirov, Gleichungen der mathematischen Physik, (Dt. Verlag d. Wissenschaften, Berlin, 1972). 